\title{
Vitamin D ameliorates neonatal necrotizing enterocolitis via suppressing TLR4 in a murine model
}

\author{
Yongyan Shi ${ }^{1,3}$, Tianjing $\mathrm{Liu}^{2,3}$, Xinyi Zhao ${ }^{1}$, Li Yao ${ }^{1}$, Ana Hou ${ }^{1}$, Jianhua $\mathrm{Fu}^{1}$ and Xindong Xue ${ }^{1}$
}

BACKGROUND: The toll-like receptor 4 (TLR4) has been reported to play an important role in necrotizing enterocolitis (NEC). As an established regulator of TLR4, vitamin D has been demonstrated to be intestinal-protective. This study aims at finding out whether the vitamin D/vitamin D receptor (VDR) pathway ameliorates NEC by regulating TLR4.

METHODS: Serum 25-hydrovitamin D (25(OH)D) was tested and compared in 15 preterm infants with NEC, 12 preterm infants without known complications and 20 healthy term infants. Neonatal Wistar rats were grouped and NEC was induced through formula feeding and cold/asphyxia stress. Vitamin D and the vehicle were administered to compare the microscopic structure, apoptotic protein expression, intestinal barrier function, inflammatory response, and TLR4 expression. RESULTS: Preterm infants with NEC had significantly lower 25 $(\mathrm{OH}) \mathrm{D}$ levels than those without NEC and healthy subjects. VDR expression was suppressed, whereas TLR4 expression was elevated in the NEC intestine. Vitamin D may increase the survival rate, alleviate structure damage, and preserve intestinal barrier function. These were achieved partly through restoration of VDR and suppression of TLR4.

CONCLUSION: NEC infants have lower levels of vitamin D. The vitamin D/NDR pathway protects against intestinal injury of NEC partly through suppressing the expression of TLR4.

W ith the increased survival rate of preterm births comes the threat of neonatal necrotizing enterocolitis (NEC). NEC affects about one in every thousand live births and $7 \%$ of the infants between 500 and1,500 g. Apart from a mortality of $20-30 \%, 25 \%$ of the NEC patients would develop short gut syndrome and neurodevelopmental impairment (1). The exact mechanism of this devastating disease remains unclear. Premature intestine, bacteria colonization, and formula feeding have been identified as contributing factors (2).

The toll-like receptor (TLR) family has been proven to be pivotal in the pathological process of NEC, especially toll-like receptor 4 (TLR4). TLR4 activation is required for the development of NEC. TLR4 mediates phagocytosis and translocation of Gram-negative bacteria. Its activation leads to increased apoptosis, reduced proliferation, and stimulated autophagy in enterocytes (3). TLR4 regulates intestinal restitution and enterocyte proliferation, both of which are vital to intestinal repair (4). TLR4 mutant mice developed less NEC (5), which had more goblet cells in the intestinal lumen and therefore produced more protective mucin (4).

A recent report disclosed some association between vitamin $\mathrm{D}$ deficiency and NEC (6). Vitamin D deficiency is prevalent in preterm births, especially in those below 32 weeks of gestation (7). As one of the regulators of TLR4, vitamin $\mathrm{D} /$ vitamin $\mathrm{D}$ receptor (VDR) pathway has been shown to be intestinal-protective by many studies. Vitamin $\mathrm{D}$ can ameliorate 2,4,6-trinitrobenzene sulfonic acid-induced colitis, a model of inflammatory bowel disease, by inhibiting intestinal epithelial apoptosis (8). In the same disease models, VDR knockout worsened the inflammatory changes, whereas human VDR transgene can significantly rescue the changes (9). Also, VDR played a suppressive role in intestinal tumor growth (10). However, the mechanism of the influence of vitamin D on NEC and the role of TLR4 has not been reported.

This study aims at: (1) investigating the vitamin D status in preterm births with and without NEC and comparing them with term subjects; (2) observing the influence of vitamin D on NEC severity in a rat model, and (3) exploring the association between vitamin D/VDR pathway and TLR4 in NEC.

\section{METHODS \\ Patients}

Among all the newborns admitted for preterm birth $(<37$ gestational week) to the department of neonatology between July and December 2012, 15 developed NEC. A total of 12 preterm infants without serious complications were selected to match the gestational week of the NEC patients. Data of 20 term births were collected in a previous study of our team (7). Caretakers of all the participants had signed written informed consent before participation. Demographic information of the participants is listed in Table 1.

\section{Patients' tissue collection}

Intestinal tissue from NEC patients was collected from those who underwent enterectomy because of irreversible necrotic changes in

\footnotetext{
'Department of Pediatrics, Shengjing Hospital of China Medical University, Shenyang, People's Republic of China; ${ }^{2}$ Department of Pediatric Orthopedics, Shengjing Hospital of China Medical University, Shenyang, People's Republic of China. Correspondence: Xindong Xue (xdxue1956@163.com)

${ }^{3}$ The first two authors contributed equally to this work.

Received 3 October 2017; accepted 18 December 2017; advance online publication 24 January 2018. doi:10.1038/pr.2017.329
} 


\section{Vitamin D suppresses TLR4 in NEC $\quad$ Articles}

Table 1. Demographic characteristics of the study population

\begin{tabular}{|c|c|c|c|c|}
\hline & Term $(n=20)$ & Preterm (no NEC) $(n=12)$ & Preterm (NEC) $(n=15)$ & $P$ value \\
\hline Gestational age (week), mean \pm SD & $39.3 \pm 1.41$ & $29.4 \pm 1.08$ & $29.3 \pm 1.16$ & $0.83^{\mathrm{a}}$ \\
\hline Birth weight $(g)$, mean $\pm S D$ & $3,279 \pm 309.6$ & $1,278 \pm 260.5$ & $1,242 \pm 235.0$ & $0.71^{\mathrm{a}}$ \\
\hline Male, $n(\%)$ & $10(50 \%)$ & $7(58.3 \%)$ & $8(53.3 \%)$ & \\
\hline Female, $n(\%)$ & $10(50 \%)$ & $5(41.7 \%)$ & $7(46.7 \%)$ & \\
\hline Season of birth, $\mathrm{n}$ (\%) & & & & 0.977 \\
\hline Spring & $4(20 \%)$ & $2(16.7 \%)$ & $3(20 \%)$ & \\
\hline Summer & $3(15 \%)$ & $3(25 \%)$ & $3(20 \%)$ & \\
\hline Autumn & $8(40 \%)$ & $5(41.7 \%)$ & $7(46.7 \%)$ & \\
\hline
\end{tabular}

NEC, necrotizing enterocolitis.

${ }^{a}$ Refers to the comparison between preterm with and without NEC, because there are sure to be significant differences between the preterm and the term babies in gestational age and birth weight.

Table 2. Demographic characteristics of the study population

\begin{tabular}{|c|c|c|c|}
\hline & NEC $(n=5)$ & INTU $(n=5)$ & $P$ value \\
\hline $\begin{array}{l}\text { Gestational age (week), } \\
\text { mean } \pm \text { SD }\end{array}$ & $29.5 \pm 1.33$ & $38.9 \pm 1.06$ & 0.000 \\
\hline Birth weight $(g)$, mean \pm SD & $1,267 \pm 257.6$ & $3,132 \pm 208.0$ & 0.000 \\
\hline Days after birth & $22.40 \pm 5.13$ & $59.8 \pm 20.46$ & 0.004 \\
\hline
\end{tabular}

intestinal segments. Demographic information of the patients is listed in Table 2. Tissue was collected randomly from the enterectomized bowel. The control groups consisted of those who received enterectomy due to irreducible intussusceptions. In this group, the tissue was harvested from the end of the enterectomized bowel away from the ischemic lesions. After harvest, the intestinal tissue was processed as mentioned below.

\section{Measurement of serum 25(OH) D levels}

As a routine test of all preterm births, blood sample was collected at the time of admittance and once a month thereafter. The sample collected at the time of admittance was used for analysis in this study. Serum $25(\mathrm{OH})$ D levels were measured by the Laboratory Test Center of Shengjing Hospital, China Medical University.

\section{Animal preparation and NEC induction}

All animal procedures were reviewed and approved by the Laboratory Animal Ethics Committee of China Medical University. This study was carried out in strict compliance with the approved protocols. Pregnant Wistar rats (200-250 g) were supplied by the Animal Lab, Experimental Research Center, Shengjing Hospital, China Medical University. All rats were kept in specific pathogenfree static cages with a 12-h light-dark cycle. Chow pellets and tap water were available ad libitum.

NEC was induced with the formula feeding and cold/asphyxia stress method. Newborn rats were separated from their mother right after delivery. They were kept warm and humidified in a newborn incubator. Neonatal animals formula was fed (Esbilac, $200 \mathrm{cal} / \mathrm{kg}$ per day; PetAg, Hampshire, IL) at $0.1 \mathrm{ml}$ every $3 \mathrm{~h}$ for $36 \mathrm{~h}$, then $0.2 \mathrm{ml}$ every $3 \mathrm{~h}$ through an orogastric feeding tube. Meanwhile, the neonatal animals were challenged with $5 \%$ oxygen for $3 \mathrm{~min}$, followed by exposure to cold $\left(4^{\circ} \mathrm{C}\right)$ for 10 min twice a day for 2 days. Neonatal rats of the control group were breastfed by their mothers and kept in room temperature and normal air.

\section{Vitamin D treatment}

The NEC and control groups were randomly divided into two subgroups. One subgroup was treated with a VD analog, paricalcitol (Sigma, St. Louis, MD, USA), dissolved in 90:10 ratio of propylene glycol:ethanol at $0.5 \mu \mathrm{g} / \mathrm{kg}$ body weight, whereas the vehicle subgroups were given the solvent only (8). Paricalcitol or vehicle was given through intraperitoneal injection $30 \mathrm{~min}$ before the first induction of NEC every day.

\section{Histology}

The distal ileum was collected and sectioned at the thickness of $4 \mu \mathrm{m}$. The slides were stained with hematoxylin and eosin (Beyotime Institute of Biotechnology, Haimen, China) at room temperature. Microscopic scoring was scored according to Nadler et al. (11). The mean score of five fields was recorded as the final score of the rat. Every group included 10 subjects. Two pathologists who were blind to the study design performed the evaluation separately.

Immunofluorescence was used to locate and evaluate occludin expression. Anti-occludin (1:100 dilution; Abcam, Cambridge, MA, USA) was used. The slides were subsequently conjugated with Alexa Fluor 555 secondary antibodies (Invitrogen, Thermo Fisher Scientific, Waltham, MA). The antigen was visualized using a Leica DFC425 fluorescence microscope (Leica Microsystems (Schweiz) AG, Heerbrugg, Switzerland).

\section{TUNEL staining}

Sections of the distal ileum were used. Terminal deoxynucleotidyl transferase-mediated dUTP nick-end labeling (TUNEL) staining was performed using an In Situ Cell Death Detection Kit, TMR red (Roche Diagnostics, Indianapolis, IN) according to the manufacturer's instructions. The apoptotic index was defined as the percentage of TUNEL-positive cell containing crypts in 100 randomly chosen crypts in each slide.

\section{Real-time PCR}

Total RNA was isolated from the distal ileum using the TRIzol reagent (Invitrogen). First-strand cDNAs were synthesised with a PrimeScript RT Reagent Kit (Takara Biotechnology, Dalian, China). Real-time PCR was performed in the $20 \mu$ l volume reaction mixture 


\section{Articles | shi et al.}

using a SYBR-Green PCR Reagent Kit (Clontech Laboratories, Mountain View, CA) and a Bio-Rad IQ5 Real-Time System (Bio-Rad laboratories, Hercules, CA, USA). The $2-\Delta \Delta \mathrm{Cq}$ formula was used to quantify the relative expression of the mRNA. $\beta-2$ Microglobulin was used as an internal control. The sequences of the PCR primers are provided in Table 3 .

\section{Western blot}

Total protein was extracted from the distal ileum. Equal amounts of proteins $(50 \mu \mathrm{g}$ per lane) were separated by $10 \%$ polyacrylamide gel electrophoresis, and the proteins were transferred electrophoretically onto polyvinylidene difluoride membranes (EMD Millipore, Billerica, MA). The membranes were then incubated with primary antibodies. The primary antibodies and their concentrations were as follows: anti-VDR (1:2,000 dilution, Santa Cruz, C20, Santa Cruz Biotechnology, Inc., Dallas, TX, USA), anti-TLR4 (1:2,000 dilution, Abcam, ab30667), anti-occludin (1:2,000 dilution, Abcam,

Table 3. Primer sequences used for real-time PCR

\begin{tabular}{|c|c|c|}
\hline $\begin{array}{l}\text { Primer } \\
\text { name }\end{array}$ & Forward $\left(5^{\prime}-3^{\prime}\right)$ & Reverse $\left(3^{\prime}-5^{\prime}\right)$ \\
\hline Rat TNF-a & ATGTGGAACTGGCA & TGGAACTGATGAG \\
\hline Rat IFN- $\gamma$ & GTGTCATCGAATCGCACCTG & GGTGACAGCTGGTGAATCAC \\
\hline Rat IL-6 & ССАCTGCCTTCCCTACTTCA & TTCTGACAGTGCATCATCGC \\
\hline Rat IL-1 $\beta$ & ACTCATTGTGGCTGTGGAGA & TAGCAGGTCGTCATCATCCC \\
\hline Rat IL-2 & CGCTTGTCCTCCTTGTCAAC & TCAATTCTGTGGCCTGCTTG \\
\hline Rat IL-17 & GAAGGCCCTCAGACTACCTC & TCAGGACCAGGATCTCTTGC \\
\hline Rat MCP-1 & GCTGCTACTCATTCACTGGC & ATTGGGC \\
\hline Rat p65 & AGCCCGTAAACTTCTCCCTC & TGTAGGCTTCACAGGACCAG \\
\hline Rat B2M & AGTGTACTCTCGCCATCCAC & CGGTGGGTGTGAATTCAGTG \\
\hline
\end{tabular}

B2M, $\beta$-2 microglobulin; IFN, interferon; IL, interleukin; MCP-1, monocyte chemoattractant protein-1; IFN, interferon; TNF, tumor necrosis factor. ab167161), anti-ZO-1 (1:2,000 dilution, Invitrogen, 339100), anticlaudin-2 (1:2,000 dilution, Abcam, ab125293), anti-PUMA (1:3,000 dilution, Cell Signaling, 7467, Cell Signaling Technology, Danvers, MA, USA), anti-caspase-3 (1:1,000 dilution, Cell Signaling, 9662), and anti- $\beta$-actin (1:2,000 dilution, Santa Cruz, sc-47778). Relative protein levels were quantified using Image J software (National Institutes of Health, Bethesda, MD).

\section{Intestinal permeability measurement}

The rats were denied access to food for $3 \mathrm{~h}$ before gavage. FITCconjugated 4-kDa dextran (FD4, $50 \mathrm{mg} / \mathrm{ml}$, Sigma) was administered via gavage at $4 \mu \mathrm{l} / \mathrm{g}$. Blood serum was collected $1 \mathrm{~h}$ later. $200 \mu \mathrm{l}$ of sample per well was added to a 96-well plate and the serum concentration of FD4 was measured using a Synergy HT plate reader (BioTek Laboratories, WA).

\section{Statistical analyses}

The data are presented as the mean \pm standard deviation. Two-tailed independent-sample $t$-test was used for comparisons between two groups. For comparisons of more than two groups, two-way ANOVA was performed (followed by Games-Howell test). Then Dunnett $t$-test was used to explore differences between selected two groups. Rank data were analyzed by the Wilcoxon's signed rank test. All statistical comparisons were done using GraphPad Prism software version 6.0 (GraphPad Software, La Jolla, CA) and SPSS 17.0 (SPSS, Chicago, IL). $P<0.05$ was regarded as statistically significant.

\section{RESULTS}

\section{Vitamin D deficiency and TLR4 elevation are observed in NEC patients}

Serum 25(OH) D concentration in the preterm infants was significantly lower than that of the term babies $(42.0 \pm 3.75$ vs. $75.6 \pm 5.29 \mathrm{nmol} / 1, P<0.05)$. Among the preterm infants, those who developed NEC had even lower 25(OH)D level a
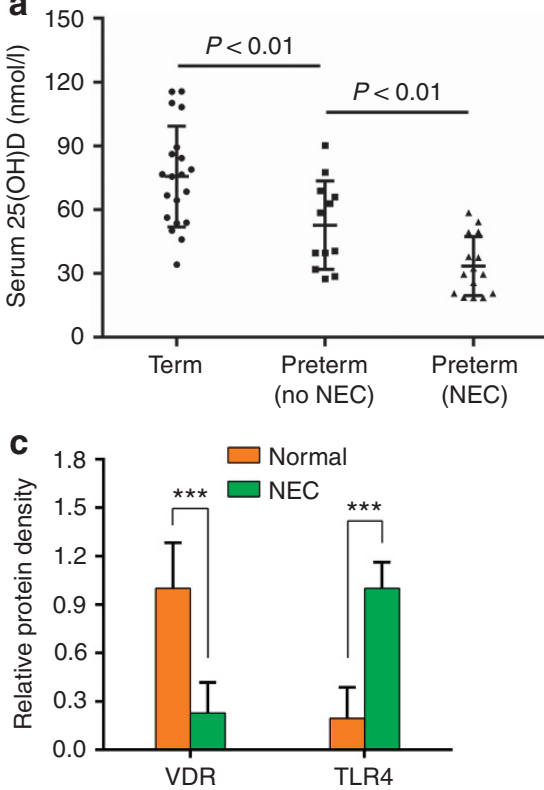

b

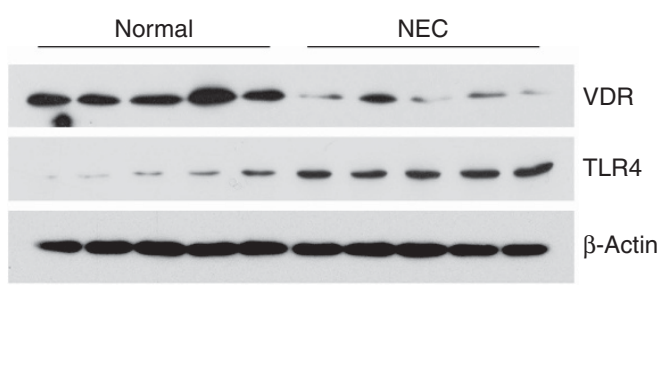

Figure 1. Comparison of vitamin $D$ level and intestinal expression of vitamin $D$ receptor (VDR) and toll-like receptor 4 (TLR4) in preterm infants with and without necrotizing enterocolitis (NEC) as well as healthy term infants, (a) serum 25-hydrovitamin D (25(OH)D) levels in each group; (b and c) western blotting showing the expression of VDR and TLR4 in normal and NEC intestines and the statistical comparisons of the relative protein density ( $n=5$ in each group). ${ }^{* *} P<0.001$. 
$(33.5 \pm 3.58$ vs. $52.7 \pm 5.99 \mathrm{nmol} / \mathrm{l})$, indicating more severe vitamin D deficiency in NEC patients (Figure 1a). Meanwhile, VDR expression was significantly diminished in intestinal tissue of NEC patients, whereas TLR4 expression was elevated (Figure 1b,c).

\section{Vitamin D can ameliorate NEC in neonatal rats}

We went on with a murine model of NEC to investigate the effect of vitamin D on NEC. NEC yielded a survival rate of $50 \%$, whereas vitamin D treatment brought the rate up to $75 \%$ (Figure 2a).

Hematoxylin and eosin staining showed intestinal structure damage, separation of the submucosa, villi discharge, or even disappearance of the villi. Spotted intestinal necrosis was also observed. Vitamin D treatment attenuated the above changes, leaving moderate aberrance of the intestinal structure (Figure 2b). NEC grade, according to Nadler, was also largely maintained by vitamin D (Figure 2c).

\section{Vitamin D attenuates apoptosis and suppresses inflammation in NEC rats}

We further looked at the mechanism of the protective function of vitamin D. TUNEL staining revealed that in NEC enterocyte apoptosis was prominent, which was greatly attenuated by vitamin D (Figure 3a). Apoptotic index confirmed this significant change (Figure $3 \mathrm{~b}$ ). Western blotting showed that the expression of apoptotic proteins, including PUMA and caspase-3, was highly activated in NEC. Vitamin D could inhibit apoptosis in both the breastfeeding and the NEC group, with the effect of the latter more significant (Figure 3c,d). Meanwhile, vitamin D treatment could suppress inflammation, as demonstrated by reduced cytokine expression in the intestine (Figure 4).

\section{Vitamin D protects against tight junction destruction and preserves intestinal barrier function in NEC}

Tight junction (TJ) proteins, as important components of the intestinal barrier, were evaluated. Western blotting showed that in NEC, TJ proteins including ZO-1, occludin, and claudin-3 decreased in expression, whereas claudin-2, a poreforming protein, increased. These changes would lead to impaired function of the intestinal barrier. Vitamin D treatment protected against this functional loss caused by NEC (Figure 5a-c). Barrier function was further evaluated through the FD4 test. Less leakage of FITC-dextran into the circulation proved that the intestinal epithelial barrier was preserved by vitamin D (Figure 5d).

\section{Vitamin D can partly rescue the VDR inhibition and TLR4 elevation in NEC}

Then we studied the changes in VDR and TLR4 expression in NEC models. In mice with NEC, the expression of TLR4 was highly elevated, whereas VDR expression was downregulated. Vitamin D could stimulate VDR expression in both the

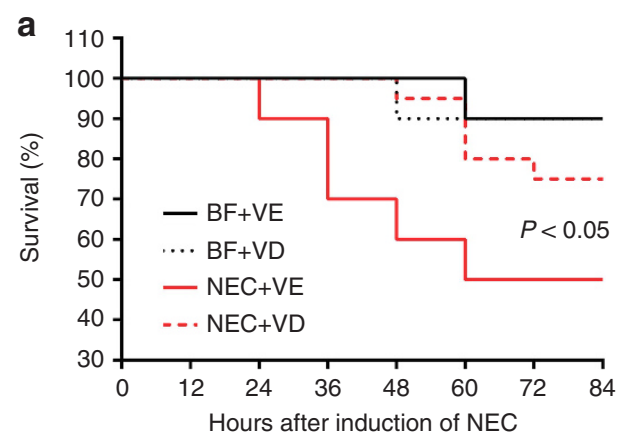

b

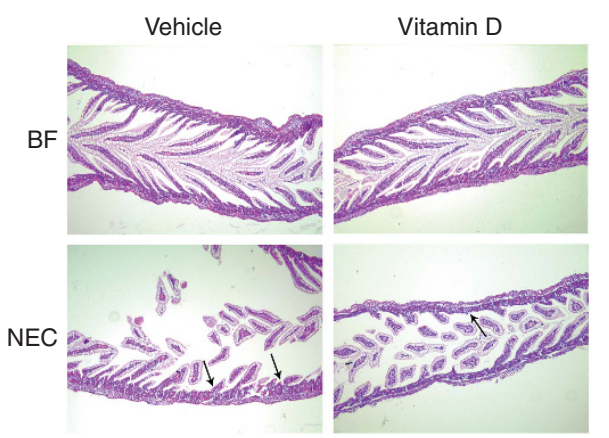

c

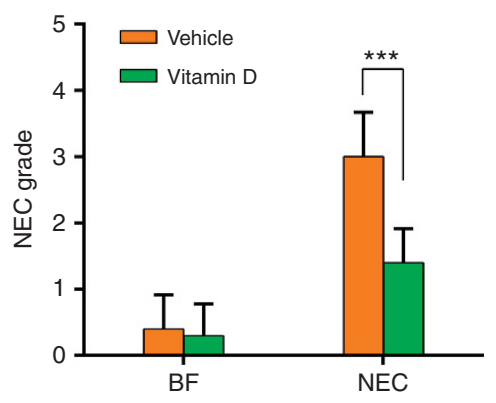

Figure 2. Vitamin D increased the survival rate and maintains structural integrity in necrotizing enterocolitis (NEC) rats. (a) Survival rate in each group ( $n=10$ in each group). (b) Hematoxylin and eosin (H\&E) staining of the intestine ( $\times 100)$. Arrows are indicating the spotted intestinal necrosis. (c) Comparison of Nadler's score, showing the severity of NEC ( $n=6$ in each group). BF, breastfeeding; BF+VE, BF+vehicle; BF+VD, BF+paricalcitol; NEC, formula feeding and cold/asphyxia stress; NEC+VD, formula feeding and cold/asphyxia stress+paricalcitol; NEC+VE, formula feeding and cold/asphyxia stress+vehicle; VD, paricalcitol; VE, vehicle. ${ }^{* * *} P<0.001$. 


\section{Articles | Shi et al.}
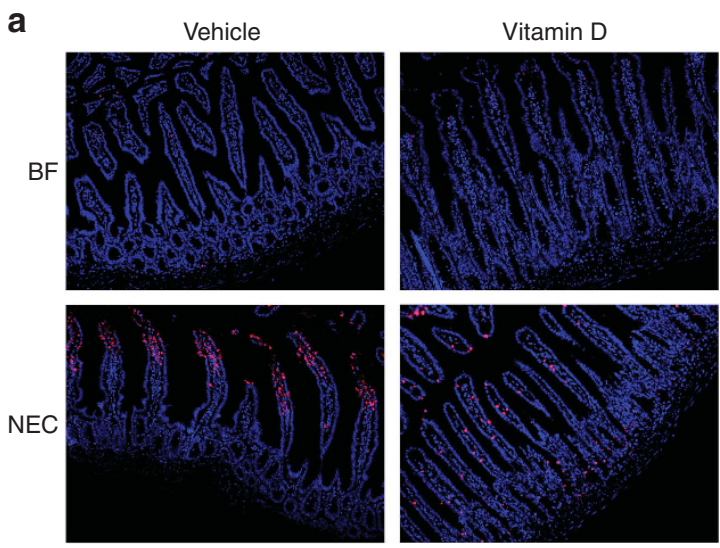

b
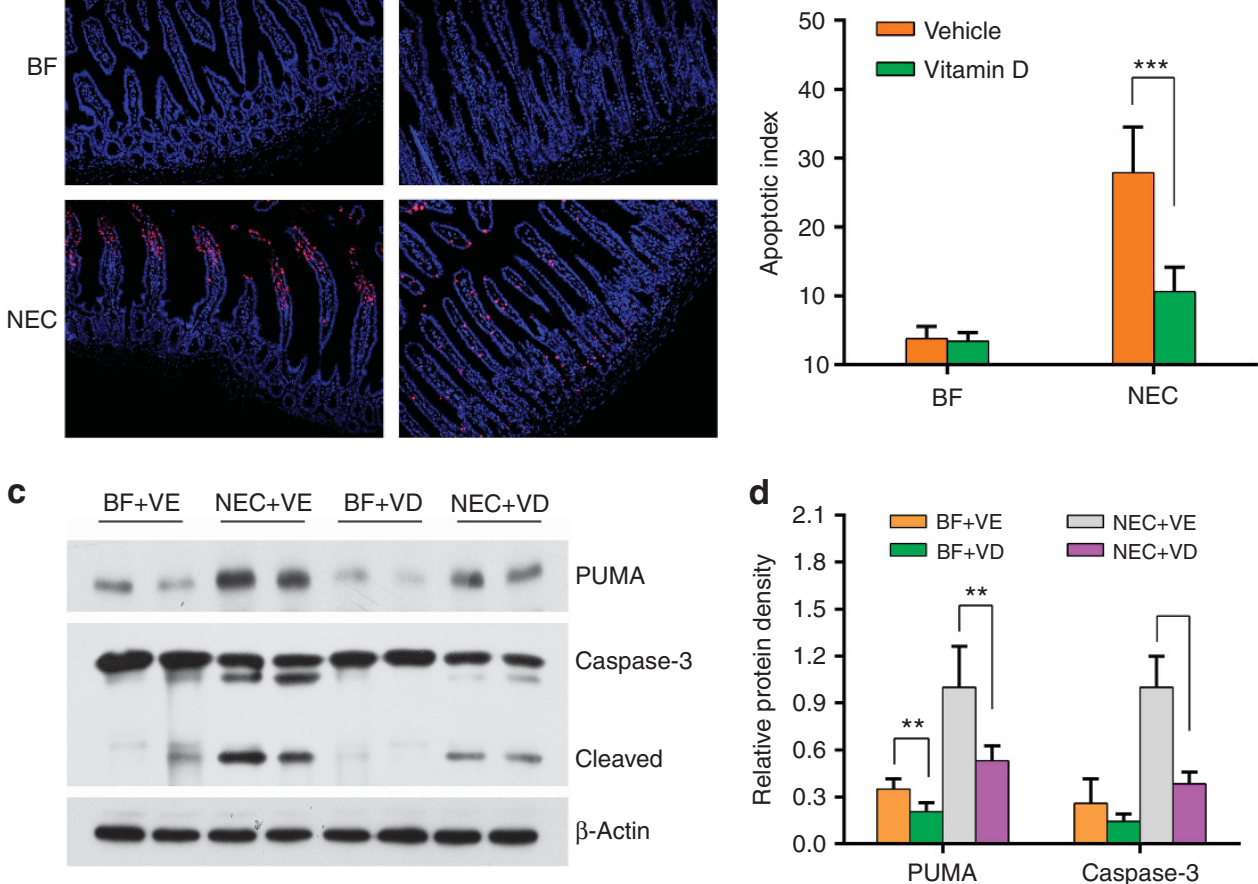

Figure 3. Vitamin D inhibited intestinal apoptosis in necrotizing enterocolitis (NEC). (a and b) Terminal deoxynucleotidyl transferase-mediated dUTP nick-end labeling (TUNEL) staining; and the apoptotic indexes ( $n=6$ in each group) $(\times 200)$. (c and d) Western blotting and relative density comparison of apoptotic proteins ( $n=6$ in each group). BF, breastfeeding; BF+VE, BF+vehicle; BF+VD, BF+paricalcitol; NEC, formula feeding and cold/asphyxia stress; NEC+VD, formula feeding and cold/asphyxia stress+paricalcitol; NEC+VE, formula feeding and cold/asphyxia stress+vehicle; VD, paricalcitol; VE, vehicle. ${ }^{* *} P<0.01 ;{ }^{* * *} P<0.001$.

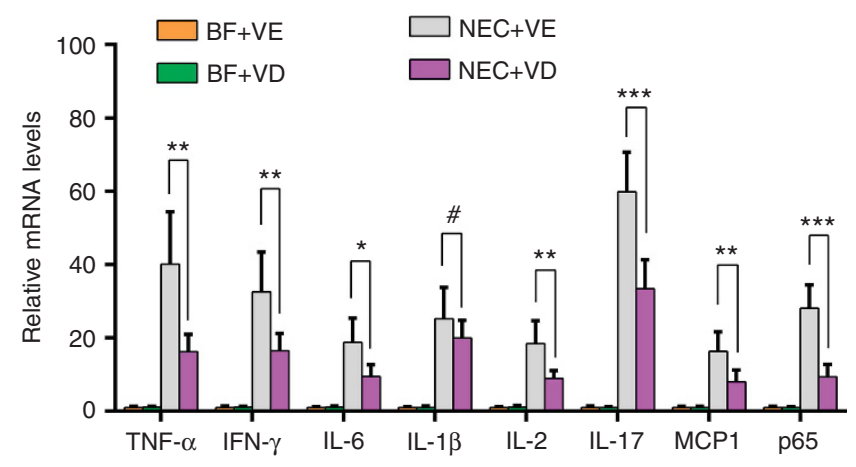

Figure 4. Cytokine mRNA expressions by real-time $P C R$. $B F+V E$, breastfeeding+vehicle; BF+VD, breastfeeding+paricalcitol; NEC, necrotizing enterocolitis; NEC+VD, formula feeding and cold/asphyxia stress+paricalcitol; NEC+VE, formula feeding and cold/asphyxia stress +vehicle. ${ }^{\#} P>0.05 ;{ }^{*} P<0.05 ;{ }^{* *} P<0.01 ;{ }^{* * *} P<0.001$.

breastfeeding and the NEC group, with the effect more prominent in the latter. The expression of TLR4 was not much influenced by vitamin $\mathrm{D}$ in healthy subjects, but was significantly rescued in the NEC rats (Figure 6).

\section{DISCUSSION}

Our study found a close association between vitamin D deficiency and NEC in preterm infants. Vitamin D could increase the survival rate, preserve intestinal structure, and maintain barrier function in a rat NEC model. Vitamin D executed antiapoptotic and anti-inflammatory effects by restoring VDR expression and downregulating TLR4 expression, which was known to be a pivotal starter of intestinal damage in NEC.

The role of TLR4 in NEC has been intensively investigated. Normally, TLR4 expression decreases after birth, whereas it increases in NEC animals. TLR4 overexpression is a typical pathological phenomenon of preterm births (1). Contributing factors of NEC, including formula feeding, hypoxia, and hypothermia, could cause TLR4 expression to increase. Another important component of NEC cascade, the plateletactivating factor, could enhance TLR4 expression (12). Inflammatory cytokines, including tumor necrosis factor and interferon, also contributed to the upregulation of TLR4 (13). When activated, TLR4 stimulates the release and activation of nuclear factor- $\mathrm{kB}$, which results in increase in proinflammatory mediators, reduced ability of spontaneous repair, and suppression of the Wnt/catenin pathway $(4,14)$. These changes had been observed both in enterocytes and intestinal stem cells (3).

Regulatory effect of vitamin D on TLR4 has been demonstrated in many organs and tissues besides the gut. Downregulation of TLR4 by vitamin D had been reported in disease models of the kidney, liver, skin, and immune cells (15-18). Our study found that the incidence of NEC in 
Vitamin D suppresses TLR4 in NEC | Articles

a

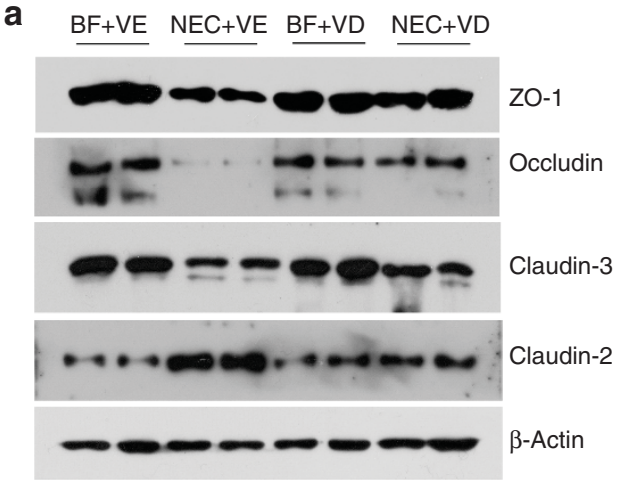

C

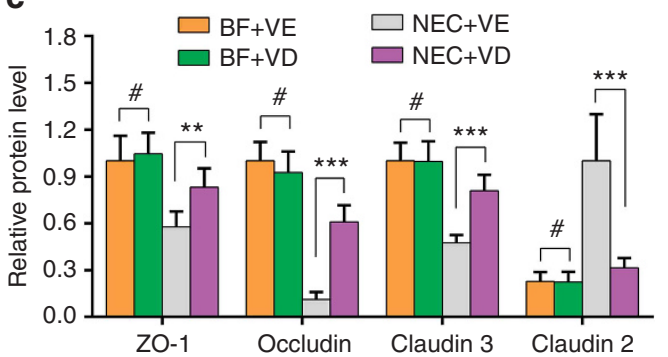

b
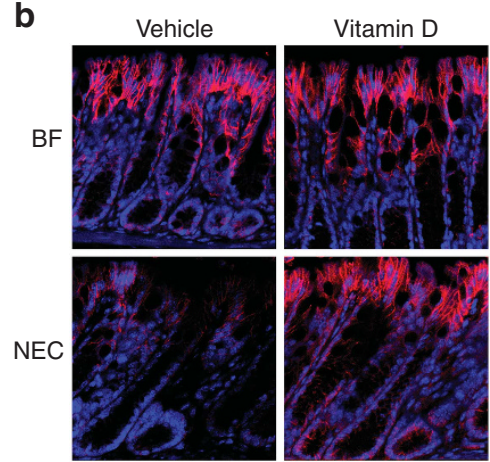

d

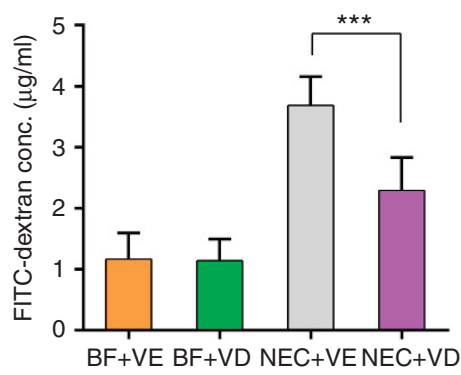

Figure 5. Tight junction proteins expression and barrier function. (a and $\mathbf{b}$ ) Western blotting and relative density comparison of tight junction proteins ( $n=6$ in each group). (c) Immunofluoresent staining of occludin. ( $\times 800)$. ${ }^{*} P>0.05 ;{ }^{* *} P<0.01 ;{ }^{* * *} P<0.001$. (d) Intestinal permeability measured by serum FITC-dextran concentration. ${ }^{* *} P<0.001$. BF, breastfeeding; $\mathrm{BF}+\mathrm{VE}$, BF+vehicle; $\mathrm{BF}+\mathrm{VD}$, $\mathrm{BF}+$ paricalcitol; $\mathrm{NEC}$, formula feeding and cold/asphyxia stress; NEC+VD, formula feeding and cold/asphyxia stress+paricalcitol; NEC+VE, formula feeding and cold/asphyxia stress+vehicle; VD, paricalcitol; VE, vehicle.

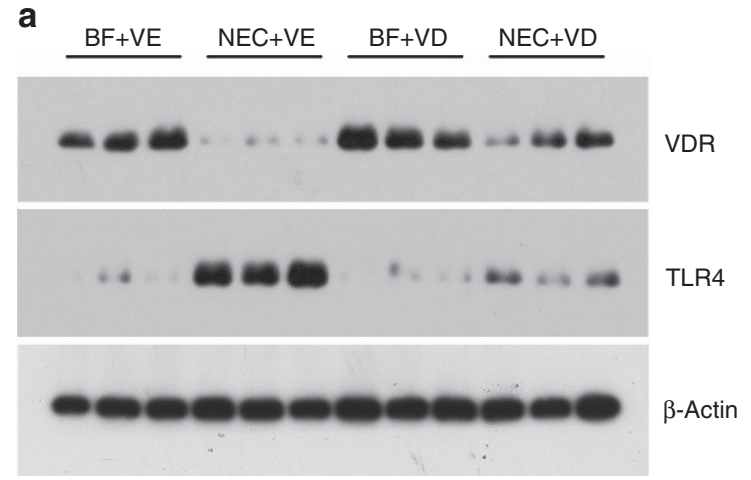

b

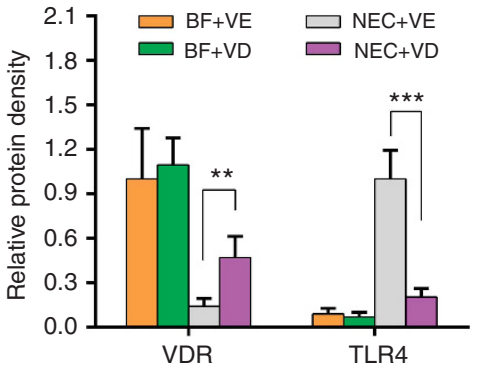

Figure 6. Western blotting showing the expression of vitamin D receptor (VDR) and toll-like receptor 4 (TLR4) and the comparison of the relative protein density. $\mathrm{BF}+\mathrm{VE}$, breastfeeding+vehicle; $\mathrm{BF}+\mathrm{VD}$, breastfeeding+paricalcitol; $\mathrm{NEC}$, necrotizing enterocolitis; $\mathrm{NEC}+\mathrm{VD}$, formula feeding and cold/ asphyxia stress+paricalcitol; NEC+VE, formula feeding and cold/asphyxia stress+vehicle. ${ }^{* *} P<0.01 ;{ }^{* * *} P<0.001$.

preterm births might be associated with vitamin D deficiency. Vitamin D pretreatment significantly suppressed TLR4 expression and therefore reduced the proinflammatory cytokine level. TUNEL staining and protein analysis revealed suppressed intestinal epithelial cell apoptosis, which was similar to the findings of Jilling et al. (19). Intestinal structure was largely preserved, as proved by milder tissue damage, less inflammatory cell infiltration, and better barrier function.

TLR4 had demonstrated immunoregulatory role in several intestinal diseases including NEC. TLR4 could mediate the influx of lymphocytes and induce NEC. By upregulating signal transducer and activator of transcription 3, TLR4 promoted the polarization of $\mathrm{T}$ cells toward $\mathrm{CD} 3^{+} \mathrm{CD} 4^{+} \mathrm{IL}-17^{+}$profile, whereas it reduced Foxp $3^{+}$regulatory T cells (20). This finding had been observed both in enterocytes and Lgr5 ${ }^{+}$intestinal stem cells (21). Our study observed elevated expression of proinflammatory cytokines in NEC, including IL-17, that could be ameliorated by vitamin $\mathrm{D}$. There might be several pathways leading to this change and inhibition of TLR4 should be one of them. The immunological mechanism of vitamin D and TLR4 on inflammation yielded further investigation.

Barrier function impairment is another important pathological change of NEC. Decrease in the expression of occludin and claudin-3 was typical (22). In this study, we detected increased 
FD4 leakage into the circulation and diminished occludin expression, indicating severe barrier injury in NEC. Vitamin D significantly attenuated these signs and preserved intestinal barrier function. The barrier-protective effect of vitamin D had previously been reported in the bowel as well as other tissue. Kong et al. (23) found that vitamin D could protect intestinal barrier in several cell lines (23). Vitamin D could restore intestinal barrier injury induced by ethanol through the redistribution of TJ proteins, lowering the phosphorylation level of myosin light chain, and decreasing the generation of ROS (24). Suppression of the nuclear factor- $\kappa \mathrm{B}$ pathway also contributed to the protective effect of vitamin $\mathrm{D}(25)$.

There might be other mechanisms of the intestinal-protective effect of vitamin D in NEC. Immunological disturbance was an important pathological basis for $\operatorname{NEC}(21,23)$, and vitamin D had long been regarded as an important immunological regulator. Thus, there was reason to believe that immunological regulation was possibly another way by which vitamin D suppressed NEC. Regulatory $\mathrm{T}$ cells, as important anti-inflammatory factors, had been reported to show an eightfold reduction in NEC (26). This decrease could be partly restored by vitamin $\mathrm{D}$ in other colitis models (27). Therefore, verification of this finding in NEC subjects was necessary to draw any conclusion.

In summary, our study found a close association between vitamin D/VDR pathway and NEC. Vitamin D may increase the survival rate, alleviate structure damage, and preserve intestinal barrier function. These were achieved partly through restoration of VDR expression and suppression of TLR4. Therefore, proinflammatory cytokines release and enterocyte apoptosis was largely inhibited. Since TLR4 activation is a typical pathological change of NEC, vitamin $\mathrm{D}$ therapy may be an effective prevention of NEC. Further clinical trials would be very helpful in confirming this finding.

\section{ACKNOWLEDGMENTS}

This study was supported by the National Nature Science Foundation of China (81700459, 81571479, and 81471489).

Disclosure: The authors declare no conflict of interest.

\section{REFERENCES}

1. Lim JC, Golden JM, Ford HR. Pathogenesis of neonatal necrotizing enterocolitis. Pediatr Surg Int 2015;31:509-18.

2. Eaton S, Rees CM, Hall NJ. Current research on the epidemiology, pathogenesis, and management of necrotizing enterocolitis. Neonatology 2017;111:423-30.

3. Meng D, Zhu W, Shi HN, et al. Toll-like receptor-4 in human and mouse colonic epithelium is developmentally regulated: a possible role in necrotizing enterocolitis. Pediatr Res 2015;77:416-24.

4. Hackam DJ, Good M, Sodhi CP. Mechanisms of gut barrier failure in the pathogenesis of necrotizing enterocolitis: Toll-like receptors throw the switch. Semin Pediatr Surg 2013;22:76-82.

5. Jilling T, Simon D, Lu J, et al. The roles of bacteria and TLR4 in rat and murine models of necrotizing enterocolitis. J Immunol 2006;177:3273-82.

6. Cetinkaya M, Erener-Ercan T, Kalayci-Oral T, et al. Maternal/neonatal vitamin D deficiency: a new risk factor for necrotizing enterocolitis in preterm infants? J Perinatol 2017;37:673-8.
7. Zhu T, Liu TJ, Ge X, Kong J, Zhang LJ, Zhao Q. High prevalence of maternal vitamin $\mathrm{D}$ deficiency in preterm births in northeast China, Shenyang. Int J Clin Exp Pathol 2015;8:1459-65.

8. Zhu T, Liu TJ, Shi YY, Zhao Q. Vitamin D/VDR signaling pathway ameliorates 2,4,6-trinitrobenzene sulfonic acid-induced colitis by inhibiting intestinal epithelial apoptosis. Int J Mol Med 2015;35:1213-8.

9. Liu W, Chen Y, Golan MA, et al. Intestinal epithelial vitamin D receptor signaling inhibits experimental colitis. J Clin Invest 2013;123:3983-96.

10. Zheng W, Wong KE, Zhang Z, et al. Inactivation of the vitamin D receptor in APCmin/+ mice reveals a critical role for the vitamin D receptor in intestinal tumor growth. Int J Cancer 2012;130:10-9.

11. Nadler EP, Dickinson E, Knisely A, et al. Expression of inducible nitric oxide synthase and interleukin-12 in experimental necrotizing enterocolitis. J Surg Res 2000;92:71-7.

12. Soliman A, Michelsen KS, Karahashi H, et al. Platelet-activating factor induces TLR4 expression in intestinal epithelial cells: implication for the pathogenesis of necrotizing enterocolitis. PLoS ONE 2010;5:e15044.

13. Caplan MS, Simon D, Jilling T. The role of PAF, TLR, and the inflammatory response in neonatal necrotizing enterocolitis. Semin Pediatr Surg 2005;14: 145-51.

14. Sodhi CP, Shi XH, Richardson WM, et al. Toll-like receptor-4 inhibits enterocyte proliferation via impaired beta-catenin signaling in necrotizing enterocolitis. Gastroenterology 2010;138:185-96.

15. Verma R, Jung JH, Kim JY. 1,25-Dihydroxyvitamin D3 up-regulates TLR10 while down-regulating TLR2, 4, and 5 in humanmonocyte THP-1. J Steroid Biochem Mol Biol 2014;141:1-6.

16. Gambhir V, Kim J, Siddiqui S, et al. Influence of 1,25-dihydroxy vitamin D3 on TLR4-induced activation of antigen presenting cells is dependent on the order of receptor engagement. Immunobiology 2011;216:988-96.

17. Lee JW, Kim SC, Ko YS, et al. Renoprotective effect of paricalcitol via a modulation of the TLR4-NF- $\mathrm{kB}$ pathway in ischemia/reperfusioninduced acute kidney injury. Biochem Biophys Res Commun 2014;444: $121-7$.

18. Jeong MS, Kim JY, Lee HI, Seo SJ. Calcitriol may down-regulate mRNA over-expression of toll-like receptor-2 and -4, LL-37 and proinflammatory cytokines in cultured human keratinocytes. Ann Dermatol 2014;26:296-302.

19. Jilling T, Lu J, Jackson M, Caplan MS. Intestinal epithelial apoptosis initiates gross bowel necrosis in an experimental rat model of neonatal necrotizing enterocolitis. Pediatr Res 2004;55:622-9.

20. Egan CE, Sodhi CP, Good M, et al. Toll-like receptor 4-mediated lymphocyte influx induces neonatal necrotizing enterocolitis. J Clin Invest. 2016;126: 495-508.

21. Niño DF, Sodhi CP, Egan CE, et al. Retinoic acid improves incidence and severity of necrotizingenterocolitis by lymphocyte balance restitution and repopulation of LGR5+ intestinal stem cells. Shock 2017;47:22-32.

22. Halpern MD, Denning PW. The role of intestinal epithelial barrier function in the development of NEC. Tissue Barriers 2015;3:e1000707.

23. Du J, Chen Y, Shi Y, et al. 1,25-Dihydroxyvitamin D protects intestinal epithelial barrier by regulating the myosin light chain kinase signaling pathway. Inflamm Bowel Dis 2015;21:2495-506.

24. Chen SW, Ma YY, Zhu J, et al. Protective effect of 1,25-dihydroxyvitamin D3 on ethanol-induced intestinal barrier injury both in vitro and in vivo. Toxicol Lett 2015;237:79-88.

25. Chen S, Zhu J, Chen G, et al. 1,25-Dihydroxyvitamin D3 preserves intestinal epithelial barrierfunction from TNF- $\alpha$ induced injury via suppression of NF-kB p65 mediated MLCK-P-MLC signaling pathway. Biochem Biophys Res Commun 2015;460:873-8.

26. Dingle BM, Liu Y, Fatheree NY, Min J, Rhoads JM, Tran DQ. FoxP3 ${ }^{+}$ regulatory $\mathrm{T}$ cells attenuate experimental necrotizing enterocolitis. PLoS ONE 2013;8:e82963.

27. Liu T, Shi Y, Du J, et al. Vitamin D treatment attenuates 2,4,6trinitrobenzene sulphonic acid (TNBS)-induced colitis but not oxazoloneinduced colitis. Sci Rep 2016;6:32889. 